\title{
HYPERBARIC OXYGEN TREATMENT IS ASSOCIATED WITH LIPID INFLAMMATORY RESPONSE ASSESSED UDING SERUM PLATELET ACTIVATING FACTOR
}

\author{
Esin Eren ${ }^{1)}$, Furkan Yıldırım²), Ozlem Giray ${ }^{3)}$, Necat Yilmaz ${ }^{1)}$ \\ 1) Health Sciences University, AEA hospital LC-MS/MS laboratory, Antalya, Turkey \\ 2) Health Sciences University, AEA hospital underwater medicine clinic, Antalya, Turkey \\ 3) Health Sciences University, AEA hospital LC-MS/MS laboratory and clinical pharmacology, Antalya, Turkey
}

\section{ABSTRACT}

Hyperbaric oxygen (HBO) treatment is generally a relatively safe therapy for various conditions. However, there are some adverse side effects. For example HBO tratment has been reported to increase the production of free oxygen radicals(FRs). Furthermore, to our knowledge, no previous clinical research has been carried out to study the involvement of platelet-activating factor(PAF)as the lipid oxidative stressor in patients undergoing HBO treatment. A total of 45 patients included in this study were first given clinical assessment and laboratory measurements before starting HBO treatment and were named group baseline. After the HBO treatment, the same clinical and laboratory measurements from the same patients were repeated and this was named group sesion $>20$.As expected, long-term HBO treatment had no effect on oxLDL (oxidized low-density lipoprotein), a lipid oxidative stress(OS) marker. However, the mean PAF values in the second group showed a statistically significant increase compared to their pretreatment values, $(\mathrm{P}<0.002)$.As this is a preliminary study, there is a need for more detailed investigations that demonstrate the association of HBO treatment with the lipid inflammatory response. Therefore, there is need for further clinical study for OS markers such as oxLDL in HBO treatment. Clinical prospective studies are required to confirm our laboratory findings. Keywords: platelet-activating factor, hyperbaric oxygen treatment, oxLDL, inflammation, oxidative stress, reactive oxygen species.

\section{ARTICLE INFO}

PolHypRes 2019 Vol. 67 Issue 2 pp. 103 - 108

ISSN: 1734-7009 eISSN: 2084-0535

DOI: $10.2478 /$ phr-2019-0009

Original article

Pages: 6, figures: 0 , tables: 0

page www of the periodical: www.phr.net.pl

Submission date: 12.03.2019 r.

Publisher

Polish Hyperbaric Medicine and Technology Society 


\section{INTRODUCTION}

Oxygen treatment has been a foundation of medical treatment for numerous pathological states. Indeed, the use of $\mathrm{O}_{2}$, mainly at supraphysiological pressures, has the potential to promote systemic oxidative stress (OS) or overproduction of $\mathrm{O}_{2}$ radicals [1]. An imbalance of free-radical formation or overconsumption of antioxidant molecules has been known to result in detrimental modification of biological macromolecules such as lipids, lipoproteins and enzymes. Indeed, OS plays an important role in the pathogenesis of diseases.

An imbalance in favor of pro-oxidants corresponds to OS and the direct action of reactive oxygen species(ROS) on cell viability and function are directly related to the occurrence of several pathological processes in the blood [2,3]. Eventually, the excessive ROS produced by OS interacts with lipids, proteins and DNA, causing structural changes, inflammation and cellular damage [4]. Whereas, under normal conditions, the production of prooxidants such as ROS is counterbalanced by antioxidants [5]. It is known that HBO threaphy also increases the production of oxygen-dependent FRs and thus has the potential to increase OS. Nevertheless, ROS have also been suggested to mediate useful actions in relation to cellular functions [6-8]. Although there are some experimental studies showing that HBO promotes antioxidant activity by providing more oxygen to the body and thus decreasing the production of ROS and lipid peroxidation, these results have not been validated [9].

Inflammatory response is a broad pathophysiological process. Numerous modern medical practices have resulted in the accumulation of an increasing number of chronic illnesses, of which many involve inflammatory responses [10]. The appropriate inflammatory response has protective effects, but overreaction often causes organ injury $[10,11]$. Primary pathological changes of inflammation include exudation, alteration and proliferation, which are mediated by inflammatory mediators such as platelet-activating factor (PAF) [12]. Many studies have shown that PAF is one of the most powerful mediators of inflammatory processes and mediates not only the inflammatory reaction induced but also the synthesis and release of other mediators to increase the degree of inflammation [12]. Evidence for PAF in atherosclerosis [11-12], cancer [13], vascular pathology [14-17], and neurodegenerative [18] processes is also presented. Thus, PAF is a potent lipid inflammatory mediator with pleiotropic effects that play a role in a variety of common diseases [12-18]. However, PAF generally plays a vital role in various physiological processes such as normal inflammatory responses, regulation of blood circulation and pressure, and regulation of coagulation [12].

In addition, PAF stimulates neutrophil chemotaxis and vascular permeability $[13,14]$. It is also accepted that PAF is partly responsible for end-organ damage in patients with systemic inflammatory response syndrome $[15,16]$. However, PAF can be thought of as a useful mediator because PAF, in general, plays a vital role in various physiological processes such as mediation of normal inflammatory responses, regulation of blood circulation and pressure, regulation of coagulation responses, glycogen degradation and neurological functions. However, PAF may be seen as an enemy because of the concern about pathological conditions related to uncontrolled inflammation, even though, at the same time, PAF is thought to be playing a role in the evolving a protective mechanism in the innate host defense system [12]. In addition, proinflammatory conditions, primarily at the basis of atherosclerotic diseases and many other diseases, are accompanied with abnormal inflammationrelated processes including PAF hyperactivity $[12,17]$. In contrast HBO treatment-related anti-inflammatory action can be induced partially through increased expression of antioxidant genes and other cellular defense genes via non-cytotoxic oxidative stimuli [18]. Although a number of studies involved PAF-producing tissues in oxygen toxicity, the exact mechanism of their pathophysiologic roles has not been fully elucidated $[19,20]$.

ROS plays an important role in lipid and lipoprotein oxidation. Therefore, oxLDL is a general term that covers heterogeneous oxidative changes at LDL. Diverse autacoid molecules of the microenvironment such as PAF and its receptor - play a significant role in the proinflammatory activation of macrophages by OS and in the uptake of oxLDL by macrophages. Because oxLDL contains inflammatory PAF-like oxidized phospholipids that mimic PAF and interact with these cells $[21,12]$.

For this reason, this study was designed to investigate the effects of HBO treatment on the lipid inflammatory mediator PAF and oxLDL levels in patients with several pathologies. Our hypothesis was that PAF and oxLDL would continue to rise with increasing oxygen pressures in contrast to anti-oxidative defense whose function would be reduced.

\section{MATERIAL AND METHOD}

\section{STUDY POPULATION AND CLINICAL EXAMINATIONS}

From January 2017 to May 2017, 45 patients (males 69\%) aged between 20 and 87 (mean: 51,1 1515,6 years), who underwent HBO treatment, were enrolled in this study prospectively. - All subjects had full physical examinations, were asked to complete a general questionnaire and gave informed consent before the commencement of the study. The questions included: age, socioeconomic status, origin of ancestors, status of physical activity, smoking, alcohol consumption, and a detailed medical history. Individuals with chronic autoimmune diseases such as Celiac disease, diabetes mellitus type 1, Graves' disease, inflammatory bowel disease, multiple sclerosis, psoriasis, rheumatoid arthritis, and systemic lupus erythematosus etc. were not included in the study.

Additionally, patients' blood pressure was measured manually with a sphygmomanometerand body mass index was calculated as weight in kilograms divided by height in meters squared. For the study, hypertension was defined as systolic blood pressure of at least 140 mmHgand diastolic blood pressure of at least $90 \mathrm{mmHg}$.

The inclusion criteria allowed patients over 18 years with no contraindications to HBO treatment. The exclusion criteria included smoking, alcohol consumption and current statin and antioxidant therapy. The demographic, clinical and historical data were recorded for all the patients. The protocol was approved by the Institutional Review Board of Antalya Training and Research Hospital and performed in accordance with the 
Declaration of Helsinki as revised in 2001. Informed consent was obtained from all participants.

\section{ApPlication OF HBO TREATMENT}

Every sequential session was conducted five days a week, and consisted of 120 minutes of $100 \%$ oxygen inhalation at 2,5 ATA (Atmosphere Absolute) with fiveminute air stopover periods every 30 minutes, in a multiplace hyperbaric chamber (Baroks $\mathrm{O}_{2}$ Multi MUL 35, Istanbul, Turkey). Each HBO treatment session also included 15-minute compression and 10-minute decompression periods.

\section{BLOOD SAMPLING AND LABORATORY ASSAYS}

Blood samples were drawn from the peripheral vein immediately before the first and directly after the 20th sessions, which represented the minimum duration of HBO treatment. In group baseline patients and group $>20$ sesion patients included in the study, after fasting at night, the blood of the patients was collected and stored in appropriate conditions and laboratory measurements were made. The blood samples were then centrifuged at 3,000 RPMs for 15 minutes, and the serum was then stored at $-80^{\circ} \mathrm{C}$ for later analysis. Lipid parameters were measured immediately.

\section{ROUTINE LABORATORY PARAMETERS}

The serum levels of triglycerides (TG), total cholesterol (TC), HDL-cholesterol (HDL-C) and serum creatinine were determined by using commercially available assay kits (Abbott) with an auto analyzer (Architect ${ }^{\circledR}$ c16000, Abbott Diagnostics, Lake Forest, Illinois, USA ). LDL cholesterol (LDL C) levels were calculated using the Friedewald formula: LDL $\mathrm{C}=$ total cholesterol - HDL - TG/2,2.

\section{MEASUREMENT OF PAF AND OXLDL LEVELS IN SERUM}

The serum samples were tested for Human PAF (Biont ${ }^{\circledR}$, Catalog no: YLA1737HU) and Human oxLDL (Biont ${ }^{\circledR}$, Catalog no:YLA0257HU) using a sandwich enzyme linked immunosorbent assay, according to the manufacturer's instructions. The results were expressed as $\mathrm{mg} / \mathrm{L}$ and $\mathrm{ng} / \mathrm{L}$, respectively.

\section{STATISTICAL ANALYSYS OF DATA}

Continuous variables were expressed as mean \pm SD, and categorical variables were presented as numbers and percentages. The statistical analysis was performed using MedCalc(C) Statistical Software version 15,8 (MedCalc Software $\AA$ bvba, Ostend, Belgium; https://www.medcalc.org; 2018). The KolmogorovSmirnov test was used to assess the distribution of continuous variables, and paired samples t-test were used to compare measurements. A p-value of $<0.05$ was considered statistically significant.

\section{RESULTS}

\section{CuINiCAl CHARACTERISTIC}

This study included 45 patients requiring HBO treatment for various pathologies. Eleven of the patients participating in the study were using antihypertensive drugs with different active ingredients. There was no statistical change in blood pressure values from before and after HBO treatment. Similarly, BMI index values of the patients did not change statistically from before and after HBO treatment. The mean body mass index was $27,1 \pm 4,4$ $\mathrm{kg} / \mathrm{m} 2$. Distribution of patients participating in the study: idiopathic sudden sensorineural hearing loss 33,3\%; diabetic foot 33,3\%; avascular necrosis 33,3\%; were incopareted. The study included 17 patients $(37,7 \%)$ with type 2 insulin dependent diabetes mellitus. There were no adverse signs observed in HBO-treated patients. At the end of HBO treatment, the success rate of the disease was 91,1\% (cured; 22,2\% and improved; 68,9\%).

Table 1, also shows the laboratory measurement results of all the patients who participated in the study. Interestingly at baseline prior to therapy, serum PAF level was $1.495 \pm 0.754 \mathrm{mg} / \mathrm{L}$. There was a statistically significant increase in PAF levels during HBO treatment in all patients. So, in the after treatment session $>20$ group, the serum PAF values of the patients treated with HBO were found to be significantly higher than the pretreatment group PAF values. (Mean \pm SD; $1.495 \pm 0.754$ $\mathrm{mg} / \mathrm{L}, \quad 1.783 \pm 0.592 \mathrm{mg} / \mathrm{L}, \mathrm{P}=0.002)$. The statistically significant increases in PAF values between baseline group and session $>20$ group was shown in table 1 and its distribution in figure 1. Similarly, oxLDL values in table 1 and oxLDL distribution in figure 2 were shown in all patients. Whereas, compared to pretreatment baseline group values (median \pm SD; $545 \pm 29 \mathrm{ng} / \mathrm{L}$ ), oxLDL levels did not change significantly after the 20th session group HBO treatment (median \pm SD; $549 \pm 33 \mathrm{ng} / \mathrm{L}$ ), $\mathrm{P}=0,45$ ). In addition, there was no statistically significant difference in routine lipid parameters after the HBO treatment in all patients.

Patients with the highest serum PAF level after HBO treatment were those with sudden sensorineural hearing loss $(1,953 \pm 0.592 \mathrm{mg} / \mathrm{l})$. In addition, a significant increase in PAF was observed in patients with diabetes and avascular necrosis at $1.75 \pm 0.53 \mathrm{mg} / \mathrm{L}$ and $1.64 \pm 0.39 \mathrm{mg} / \mathrm{l}$ respectively. However, there was no statistically significant difference in these increases in serum PAF levels between the different pathological groups after the HBO treatment $(\mathrm{p}<0.05)$.

\section{DISCUSSION}

First, HBO treatment may increase the in vitro lipid mediator(s), preferably induced by increased levels of serum PAF. The exact mechanism for the undesired response of PAF in patients receiving HBO treatment has not yet been fully determined in the literature.

Metabolic changes after HBO treatment, especially focused on oxLDL, have not been fully described in humans. Many of the researchers investigating the effect of HBO treatment on antioxidant enzymes in experimental animals have published different results [22,23]. Theoretically, oxLDL is a reliable indicator of OS, and is one of the products of HBO treatment. However, similar to previous studies, there was no statistically significant difference in serum oxLDL levels after HBO treatment in our study, which is in accordance with previous studies [22,23]. 
It seems oxLDL was not involved in the increase in PAF-induced inflammatory responses caused by HBO treatment. In addition, other routine blood lipid parameters did not seem to be affected by prolonged HBO treatment. In fact, with long-term and repeated administration, HBO treatment below 2,0 Absolute Atmosphere can actually reduce oxidative stress by reducing lipid peroxidation and by upregulating the activity of many antioxidant enzymes [24, 25]. However, in animal studies, many important positive antiinflammatory effects of both normobaric and hyperbaric oxygen have been repeatedly shown [24].

To our knowledge, in vivo human lipid inflammatory mediator PAF measurement and relations of hyperbaric treatment have not been previously shown in medical literature. The uniqueness of this study lies in the measurement of whole-body lipid inflammatory stress using a reliable biomarker by PAF sensitive to changes in oxygen concentration. This measurement(s) may be important in an environment where patients are exposed to extremely high concentrations of oxygen, such as during HBO treatment. Moreover, PAF administration during HBO treatment, as shown in a previous study, may activate white blood cells and improve ROS production $[19,20]$. Indeed some previous studies reported that for intracellular antioxidant enzymes, the catalase and glutathione peroxidase were decreased by PAF administration [19].

These data support the hypothesis that serum PAF can be affected by the presence of oxygen in the environment. However, to date, there are few studies investigating the occurrence of PAF during HBO treatment in patients. Indeed, in investigating the presence and amount of inflammation that may occur during HBO therapy, some biomarkers, each of which exhibits several advantages and disadvantages, have been investigated [12, 25]. This study showed the effect of the interaction between HBO treatment and PAF on inflammatory response in vivo. This is the first report demonstrating increased PAF levels in patients during HBO treatment, suggesting that HBO therapies are a previously unrecognized source of lipid mediator in inflammatory processes.

Normally, plasma and tissue levels of PAF are tightly regulated by its metabolic pathways. PAF is a bioactive lipid produced by a wide range of cells in various tissues [12]. Thus, its synthesis, distribution and degradation are all strictly controlled, as would be expected for such a potent molecule with a wide range of diverse actions. The basal PAF source in the plasma is the kidney. Other tissues where PAF is synthesized include the liver, spleen and the cells of the blood are macrophages $[12,15]$. During inflammation, and in many cells with a role in allergies, eg neutrophils, when they are activated, PAF is synthesized and released [15]. Another source of PAF is human endothelial cells. So, PAF is a potent lipid mediator that is implicated in numerous inflammatory diseases. PAF acts on several receptors on cellular and nuclear membranes. Under normal circumstances, homeostatic levels of PAF present in plasma and biological tissue seem to be regulated by a balance of its biosynthetic and catabolic enzymatic pathways [12]. Presumably, inflammatory responses can be increased by decreased antioxidant response. Perhaps the oxygen present in the environment during HBO therapy may lead to PAF synthesis. In addition to enzymatic biosynthetic pathways, PAF can also be produced by non-enzymatic synthesis through the oxidation of lipids during OS [12].

The production of PAF and such PAF-like oxidized lipids usually occurs during inflammation and oxidative stress-related diseases. In addition, it is known that PAF increase vascular permeability. Thus, PAF can further propagate OS through the oxidation of LDL and the reduction of NO bioavailability [12]. Some researchers have suggested that chronic and intermittent treatment with HBO treatment can induce components of the antioxidant defense response and may even lead to a decrease in the level of oxLDL, which may be atheroprotective [25].

Conversely, several studies previously reported that there was no significant difference in the erythrocyte antioxidant capacity or lipid peroxidation by HBO treatment [12]. Finally, no studies have looked for serum PAF level changes during HBO treatment in humans. Based on our findings, it appears that the accumulation of PAF raised in correlation to and in direct response to the number of HBO treatment sessions. HBO treatment is generally a relatively safe treatment for various medical conditions. Nowadays, it is reported that HBO treatment may have side effects. Because hyperexia can be as harmful as hypoxia, careful application of trreatment is required. [19].

Usual junctions, such as PAF and its related inflammatory pathways, seem to be encouraging therapeutic targets for the prevention and treatment of the onset and progression of inflammation-related chronic diseases. Patients treated with HBO may use a PAF inhibitor (for example: Rhodium(I-III) Compounds) or a PAF-reducing diet (for example; Ginkgo biloba, Mediterranean diet etc.) as protection against lipid inflammation. (12). In fact, up to date; there is no evidence that PAF receptor antagonist administration might attenuate the inflammation created by HBO treatment. Chronic inflammation can cause endothelial dysfunction. Ultimately, the basic molecular mechanism of diseases such as atherosclerosis and cancer involves endothelial dysfunction [25]. PAF may play a role in the physiological control of blood pressure since the factors affecting renal function may alter the plasma levels of PAF [26].

This preliminary manuscript has some limitations. The small number of patients included in this study is an important deficiency. In addition, patients with HBO have very different pathology, making it difficult to comment. There is a need for investigating the cause of increase in serum PAF levels after HBO treatment and studies involving more homogeneous patient groups.

Ultimately, in this preliminary study, we observed that increased PAF levels caused by HBO treatment may have affected the inflammatory response by not including oxLDL production, and exposure to HBO may have enhanced this effect. Further research is needed to understand the mechanisms underlying the observed associations. 


\section{REFERENCES}

1. Sjöberg F, Singer M. The medical use of oxygen: a time for critical reappraisal. J Intern Med. 2013 Dec;274(6):505-28. doi: 10.1111/joim.12139;

2. Yilmaz N. Relationship between paraoxonase and homocysteine: crossroads of oxidative diseases. Arch Med Sci. 2012 Feb 29;8(1):138-53. doi: 10.5114/aoms.2012.27294;

3. Allen MW, Golembe E, Gorenstein S, Butler GJ. Protective effects of hyperbaric oxygen therapy (HBO2) in cardiac care-a proposal to conduct a study into the effects of hyperbaric preconditioning in elective coronary artery bypass graft surgery (CABG). Undersea Hyperb Med.2015 MarApr; 42.107-114. doi:10.2214/ajr.151.5.1003;

4. Davalli P, Mitic T, Caporali A, Lauriola A, D'Arca D. ROS, cell senescence, and novel molecular mechanisms in aging and age-related diseases Oxid Med Cell Longev. 2016;2016:3565127. doi: 10.1155/2016/3565127;

5. Rahal A, Kumar A, Singh V et al. Oxidative stress, prooxidants, and antioxidants: the interplay. Biomed Res Int. 2014;2014:761264. doi: 10.1155/2014/761264;

6. Dennog C, Hartmann A, Frey G, Speit G. Detection of DNA damage after hyperbaric oxygen (HBO2) therapy. Mutagenesis. 1996 Nov;11(6):605609. doi:10.10193.

7. Narkowicz CK, Vial JH, McCartney PW. Hyperbaric oxygen therapy increases free radical levels in the blood of humans. Free Radic Res Commun. 1993;19(2):71-80. PMID: 8225040 doi:10.3109/10715769309056501;

8. Simsek K, Ozler M, Ucar E, et al. Pressure-related effects of hyperbaric oxygen exposure on oxidation products and antioxidant enzymes in the rat lung. J Exp Integr Med. 2011; 1(1):37-42 doi:10.5455/jeim.130111.or.003.

9. Simsek K, Ozler M, Yildirim AO et al. Evaluation of the oxidative effect of long term repetitive hyperbaric oxygen exposures on different brain regions of rats. Scientific World Journal. 2012;2012:849183. doi:10.1100/2012/849183;

10. Eren E, Ellidag HY, Aydin O, Yilmaz N. HDL functionality and crystal-based sterile inflammation in atherosclerosis. Clin Chim Acta. 2015 Jan 15;439,18-23. doi: 10.1016/j.cca.2014.09.024;

11. McGarry T, Biniecka M, Veale DJ, Fearon U. Hypoxia, oxidative stress and inflammation. Free Radic Biol Med. 2018 Mar 27. pii: S08915849(18)30145-X. doi: 10.1016/j.freeradbiomed.2018.03.042;

12. Tsoupras A, Lordan R, Zabetakis I. Inflammation, not cholesterol, is a cause of chronic disease. Nutrients. 2018 May 12;10(5). pii: E604. doi: 10.3390/nu10050604

13. Tsoupras $A B$, latrou $C$, Frangia $C$, Demopoulos $C A$. The implication of platelet activating factor in cancer growth and metastasis: potent beneficia role of PAF-inhibitors and antioxidants. Infect Disord Drug Targets. 2009 Aug;9(4):390-9. doi:10.2174/187152609788922555;

14. Palur Ramakrishnan AV, Varghese TP, Vanapalli S, Nair NK, Mingate MD. Platelet activating factor: A potential biomarker in acute coronary syndrome? Cardiovasc Ther. 2017 Feb;35(1):64-70. doi: 10.1111/1755-5922.12233

15. Nakamura T, Murata T. Regulation of vascular permeability in anaphylaxis. Br J Pharmacol. 2018 Jul;175(13):2538-2542 doi: 10.1111/bph.14332

16. Bulger EM, Maier RV. Lipid mediators in the pathophysiology of critical illness. Crit Care Med. 2000 Apr;28(4 Suppl):N27-36 doi:10.1097/00003246-200004001-00004

17. Papakonstantinou VD, Lagopati N, Tsilibary EC, Demopoulos CA, Philippopoulos AI. A review on platelet activating factor inhibitors: could a new class of potent metal-based anti-inflammatory drugs induce anticancer properties? Bioinorg Chem Appl. 2017;2017:6947034, doi: $10.1155 / 2017 / 6947034$

18. Gao ZX, Rao J, Li YH. Hyperbaric oxygen preconditioning improves postoperative cognitive dysfunction by reducing oxidant stress and inflammation. Neural Regen Res. 2017 Feb;12(2):329-336 doi: 10.4103/1673-5374.200816;

19. Yildirim G, Baştuğ M, Akçil IE, Fiçicilar H, Koç E, Zaloğlu N. Does the plateletactivating factor affect the antioxidant defense system? The possible role of hyperbaric oxygenation. Biol Trace Elem Res. 2000 Winter;78(1-3):7-12. doi:10,1385/BTER:78:1-3:7;

20. Yildirim G, Koç E, Baştug M, Fiçicilar H, Akçil IE, Zaloglu N. Effects of plateletactivating factor and hyperbaric oxygenation on antioxidant defense and duodenal contractility. Undersea Hyperb Med. 2000 Fall;27(3):155-8.doi.org/10.22462/01.02.2018.6;

21. Eren E, Yilmaz N, Aydin O. High density lipoprotein and it's dysfunction. Open Biochem J. 2012;6.78-93 doi: 10,2174/1874091X01206010078;

22. Keskin K, Kilci H, Aksan G, et al. Serum oxidized low-density lipoprotein level as a marker of oxidative stress in patients undergoing hyperbaric oxygen therapy. Turk Kardiyol Dern Ars. 2017 Sep;45(6):533-537. doi: 10.5543/tkda.2017.28302;

23. Matzi V, Greilberger JF, Lindenmann J, et al. Application of hyperbaric oxygen reduce oxidative damage of plasmatic carbonyl proteins and 8OHdG by activating glutathion peroxidase. Clin Lab. 2015;61(5-6):587-93.doi:10.7754/Clin.Lab.2014.140929;

24. Kudchodkar B, Jones H, Simecka J, Dory L. Hyperbaric oxygen treatment attenuates the pro-inflammatory and immune responses in apolipoprotein E knockout mice. Clin Immunol. 2008 Sep;128(3):435-41. doi: 10.1016/j.clim.2008.05.004; Epub 2008 Jul 2 ;

25. Kudchodkar BJ, Pierce A, Dory L. Chronic hyperbaric oxygen treatment elicits an antioxidant response and attenuates atherosclerosis in apoE knockout mice. Atherosclerosis. 2007 Jul;193(1):28-35. doi:10.1016/j.atherosclerosis.2006.08.018;

26. Snyder F.Platelet-activating factor and related acetylated lipids as potent biologically active cellular mediators. Am J Physiol. $1990 \mathrm{Nov} ; 259$ (5 Pt 1):C697-708.doi:10.1152/ajpcell.1990.259.5.C697;

Prof dr Necat Yilmaz SBÜ

AEAH Lc-MS/MS laboratory clinical laboratory education director Antalya, Turkey

e-mail: necatyilmaz@hotmail.com 